\section{Response to 'Low-dose versus high-dose fish oil for pain reduction and function improvement in patients with knee osteoarthritis' by Chen et al}

We thank Chen et $a l^{1}$ for their interest in our paper ${ }^{2}$ and are pleased to have the opportunity to respond to their comments.

Our study was a multicentre, double-blind, randomised control trial designed specifically to answer the question whether high-dose fish oil was superior to low-dose fish oil for treatment of knee osteoarthritis, and our findings were that it was not. ${ }^{2}$ This question arose from the important work of our colleagues and coauthors in demonstrating anti-inflammatory effects of high-dose fish oil and associated treatment benefits in patients with rheumatoid arthritis. ${ }^{3}$ Therefore, the rationale and underlying hypothesis for the study in osteoarthritis specifically related to anti-inflammatory properties of high-dose fish oil, and there was no prior rationale for proposing that low-dose fish oil may also be of benefit. WOMAC pain and function improved in both treatment groups during the course of the trial. However, as substantial regression to the mean effects is observed with these outcomes in osteoarthritis trials, including our study, it is not possible to determine, as Chen et al have pointed out, whether low-dose fish oil may in fact be of some benefit for osteoarthritis. An equally valid question is whether sunola oil, the diluent used in the low-dose group, may also be of therapeutic benefit. However, at study inception, we considered low-dose fish oil plus sunola to be a placebo based on existing knowledge at the time. These are question of the possible benefit of sunola oil is for future research, and it is difficult to decide, even with the benefit of hindsight, whether we should have included a placebo arm, given the ethical issues and problems with blinding this would have entailed.

Chen et al have criticised the lack of detail in relation to patient recruitment in our paper, yet we provided extensive information about the patient recruitment and the complete study protocol in the online supplementary material. Like all clinical trials, our study had both withdrawals from therapy and losses to follow-up, yet we do not believe this was sufficient to suggest bias. We collected data at six treatment visits over 2 years, obtained data from $>80 \%$ of the participants at the last study visit, and used multiply imputed datasets for an intention-to-treat longitudinal data analysis.

Chen et al have also criticised our choice of outcome measures. Our primary outcome was WOMAC pain, and we stand by that as the most appropriate, patient-centred outcome measure, given our underlying hypothesis. Secondary outcome measures included WOMAC function, quality of life, hsCRP and cartilage volume and bone marrow lesions assessed by MRI. In the absence of any demonstrable benefit of high-dose fish oil for any of the highly relevant outcome measures employed, it is not clear what the additional secondary outcome measures proposed by Chen et al would have contributed to the study. We are able to measure effusion within our dataset. However, this has never been tested as an outcome measure in clinical trials, and will be used for hypothesis generation.

Other issues raised were with respect to potential confounders such as other musculoskeletal diseases, comorbidities, exercise and alcohol consumption. This of course is exactly why the study participants were randomised. Finally, we disagree that adverse events were not properly reported. In fact, the number of adverse events seems high because we meticulously recorded, collated and reported every adverse event reported by these middle-aged to elderly participants at each of the six treatment visits over 2 years, whether or not these events required hospitalisation, or could potentially be ascribed to fish oil treatment. However, we did note that gastrointestinal bleeding and cardiovascular events were comparable between the two treatment groups.

In summary, we believe our study was a rigorous, welldesigned and well-conducted randomised controlled trial, which clearly demonstrates that high-dose fish oil is not superior to low-dose fish oil in knee osteoarthritis.

\section{Catherine Hill, ${ }^{1,2}$ Susan Elizabeth Lester, ${ }^{3}$ Graeme Jones ${ }^{4}$}

${ }^{1}$ Department of Rheumatology, The Queen Elizabeth Hospital, Woodville, South Australia, Australia

${ }^{2}$ The Health Observatory, University of Adelaide, Adelaide, South Australia, Australia ${ }^{3}$ Department of Rheumatology, The Queen Elizabeth Hospital, Adelaide, South Australia, Australia

${ }^{4}$ Hobart, Tasmania, Australia

Correspondence to Dr Catherine Hill, Department of Rheumatology, The Queen Elizabeth Hospital, Woodville, SA 5011, Australia; catherine.hill@health.sa.gov.au

Competing interests None declared.

Provenance and peer review Commissioned; internally peer reviewed.

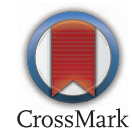

To cite Hill C, Lester SE, Jones G. Ann Rheum Dis 2016;75:e8.

Accepted 2 November 2015

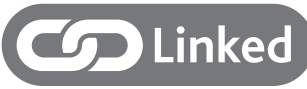

- http://dx.doi.org/10.1136/annrheumdis-2015-208754

Ann Rheum Dis 2016;75:e8. doi:10.1136/annrheumdis-2015-208781

\section{REFERENCES}

1 Chen Y, Huang Y-C, Lu WW. Low-dose versus high-dose fish oil for pain reduction and function improvement in patients with knee osteoarthritis. Ann Rheum Dis 2016;75:e7.

2 Hill CL, March LM, Aitken D, et al. Fish oil in knee osteoarthritis: a randomised clinical trial of low dose versus high dose. Ann Rheum Dis 2016;75:23-9.

3 Proudman SM, James MJ, Spargo LD, et al. Fish oil in recent onset rheumatoid arthritis: a randomised, double-blind controlled trial within algorithm-based drug use. Ann Rheum Dis 2015;74:89-95. 